

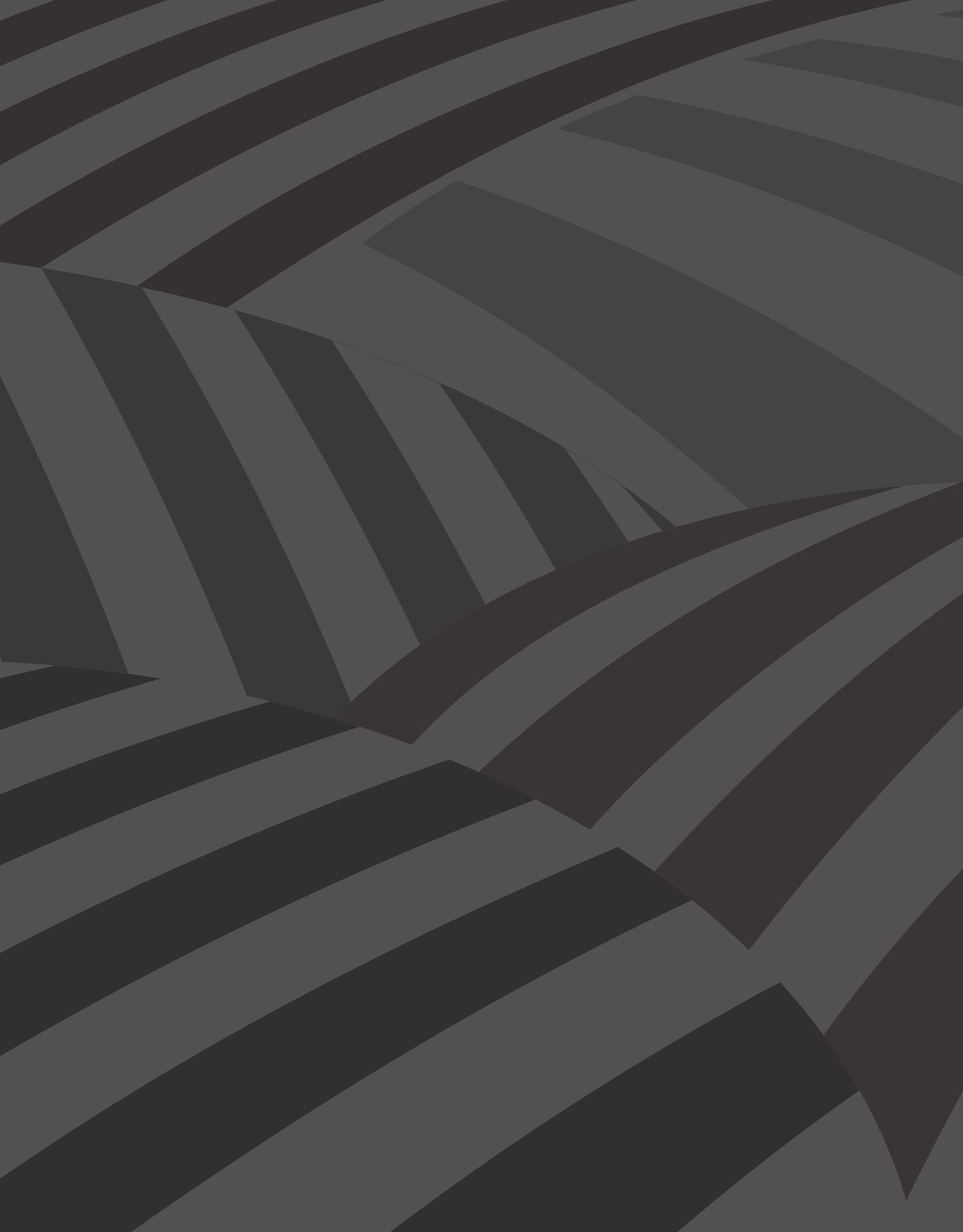




\title{
Los Principios Ruggie y la debida diligencia en el derecho fundamental de Consulta Previa
}

\author{
Por Piedad Andrea Rodríguez*, Daniela Alejandra Gómez ${ }^{* *}$ y Daniela Caicedo***
}

Resumen: En este artículo se aborda la relación entre empresa y Estado, que se encuentra ligada por la aplicación de los Principios Rectores de las Naciones Unidas sobre las empresas y los derechos humanos «John Ruggie». Aunque estos principios no son fuente formal del derecho internacional, ya que son soft law, en su esencia, recopilan las bases para mitigar, prevenir y reparar las afectaciones a derechos humanos que causen las empresas en el desarrollo de actividades económicas a comunidades que se encuentren en un territorio específico. Debido a esto, cada vez más se ve su aplicación en políticas públicas por parte de los Estados y en planes de ejecución empresarial mediante la aplicación de la debida diligencia. Este concepto cobra mayor relevancia por ser una herramienta más de defensa de la Consulta Previa, y a su vez ser el puente de conexión entre la aplicación de los Principios Rectores y los efectos que conlleva generar una afectación a los derechos humanos.

Palabras clave: Debida Diligencia, Principios Rectores de Naciones Unidas (PRNU), consulta previa, comunidades, Estado, empresa, Derechos Humanos (DD. HH.).

\section{Ruggie Principles: Due diligence and Prior Consultation}

Abstract: This article approach the relationship between business and the State is linked by the application of the United Nations Guiding Principles on Human Rights and Business «John Ruggie». Although these principles are not a formal source of international law since they are soft law, in essence, they gather the bases to mitigate, prevent and repair the human rights violations caused by companies in the development of economic activities to communities that are in a specific territory. Also, it is increasingly seen its application in public policies by the States and in business

* Abogada de la Universidad Javeriana de Cali. Investigadora de la línea de Desarrollo Rural y Ordenamiento Territorial del Instituto de Estudios Interculturales (IEI) de la Universidad Javeriana de Cali. Directora del Semillero de Arbitraje Internacional y docente del Programa de Derecho de la Universidad Javeriana de Cali

** Socióloga y abogada de la Universidad Icesi. Investigadora de la línea de Desarrollo Rural y Ordenamiento Territorial del Instituto de Estudios Interculturales (IEI) de la Universidad Javeriana de Cali

*** Socióloga y antropóloga de la Universidad Icesi de Cali. Investigadora de la línea de Desarrollo Rural y Ordenamiento Territorial del Instituto de Estudios Interculturales (IEI) de la Universidad Javeriana de Cali. 
execution plans through the application of due diligence. This concept is more relevant because it is one more tool to defend the Prior Consultation; and at the same time, be the connecting bridge between the application of the Guiding Principles and the effects entailed in generating an impact on human rights.

Keywords: Due Diligense, United Nations Guiding Principles on Human Rights and Business, community, State, company, Human Right.

Cómo citar este artículo: Rodríguez, Piedad Andrea; Gómez, Daniela Alejandra y Caicedo, Daniela (2019). Los Principios Ruggie y la debida diligencia en el derecho fundamental de Consulta Previa. Revista Controversia, 212, 49-66.

Fecha de recepción: 9 de diciembre de 2018

Fecha de aprobación: 28 de febrero de 2019

\section{La relación entre los derechos humanos (DD. HH.) y la empresa}

T a Declaración Universal de los Derechos Humanos (DD. HH.) se constituyó como un acuerdo que era acogido y adoptado únicamente por algunos Estados. Inicialmente se pensó que esta figura era la única involucrada en procesos que pudieran afectar a las comunidades y a la sociedad en general. Desde la década de 1970 se iniciaron las primeras apuestas por establecer normas internacionales que se aplicaran al contexto empresarial. De hecho, en 1973 se estableció la Comisión de Naciones Unidas sobre Empresas Transnacionales que emprendió el proyecto de Código de Conducta de Naciones Unidas para Empresas Transnacionales, interesado en regular social y ambientalmente a las empresas (Hurtado, 2011, p. 7). Seguido de esto, en 1976 la Organización para la Cooperación Económica y el Desarrollo (OCDE) estableció las Directrices para Empresas Multinacionales sin tener mayor impacto. Luego, en 1977, la Organización Internacional del Trabajo (OIT) adoptó la Declaración Tripartita de Principios sobre las Empresas Multinacionales en la que se abordó por primera vez el tema de los derechos laborales en el marco de los derechos humanos (Hurtado, 2011, 
p. 7). Pero, hasta ese momento no lograba posicionarse en el panorama ninguna discusión que proyectara seriamente la regulación de las acciones de las empresas y su posible responsabilidad en las afectaciones que pudiera tener la sociedad civil.

En este sentido, ya en la década de 1990 empezó con mayor fuerza la discusión en torno a la existencia de actores que con su influencia y su capacidad de acción, podrían convertirse en figuras responsables de daños o perjuicios. De manera más directa, el papel de las empresas, en su relación con las comunidades y la sociedad en general, empezó a cuestionarse a nivel internacional luego de eventos como el accidente en la fábrica de pesticidas en Bhopal, en 1984, considerada la catástrofe industrial más grande de la historia, que ocasionó la muerte de 25 000 personas y aún, hasta la fecha, hay nuevas generaciones afectadas (I Arrufat, 2014). De igual forma, en 1996, la fundación Made in Usa acusó a la empresa de calzado Nike de contratar niños de 11 años en sus plantas de producción en Indonesia, y pagarles 14 centavos al día (Calvo, 1996). Estas situaciones pusieron en el ojo de la opinión pública la relación entre empresa y derechos humanos, así como también se cuestionó el proceso de reparación e indemnización que ya dejaba de ser una responsabilidad directa del Estado.

Por esta razón, y debido a las nuevas lógicas empresariales a nivel mundial y su injerencia en la apertura económica de las naciones, las empresas empezaron a considerarse como actores que también podrían violar derechos humanos y que debían asumir esa responsabilidad. De modo que surgió una segunda ola de interés en el reconocimiento de un actor distinto al Estado que, si bien había gozado históricamente de una suerte de independencia con respecto a la regulación, ya tendría mayor supervisión en sus acciones.

Como es ampliamente sabido, en América Latina, en lo que va de este siglo, se ha incrementado la actividad extractiva. Esto implica, la mayoría de las veces, la implementación de proyectos en territorios 
ubicados en zonas periféricas, donde el acceso del Estado, e incluso su interlocución, es fragmentaria (Slack, 2011, p. 4). En su mayoría, estos proyectos han incurrido en la no realización de consultas adecuadas a las poblaciones aledañas y posiblemente afectadas, pero también se han visto involucradas en episodios de violencia. En este contexto, la movilización social y los procesos de resistencia de estas comunidades son cada vez más cercanos a la opinión pública, y ponen, con mayor frecuencia, en evidencia los retos que enfrentan constantemente con la dependencia económica de la extracción de minerales y los modelos de desarrollo sostenible. Es importante que se promueva la mitigación de los daños causados al medioambiente y a las comunidades, a través de las herramientas que históricamente el derecho internacional ha consolidado e incluso a través del fortalecimiento de los mecanismos internos de protección. Por lo tanto, es necesaria la formulación y el cumplimiento de acuerdos que regulen la protección de los derechos humanos en los contextos proclives a su violación.

Al parecer, en este contexto, la capacidad de los Estados para supervisar las relaciones que las empresas establecen con las comunidades es reducida, por lo que en ocasiones estas interacciones se dan en escenarios de escasa formalidad e intermediación. De hecho, este es uno de los primeros escenarios de violación a los derechos humanos y al mismo tiempo uno de los más habituales. Pero esto se ha convertido en un patrón que durante los últimos años ha engrandecido las luchas de las organizaciones sociales y ha puesto en tela de juicio la capacidad de los Estados para impartir justicia. No obstante, como se presentará a continuación, actualmente existe una reglamentación que está empezando a ser reconocida y ejercida por las comunidades y los Estados para exigir la protección de sus derechos fundamentales en contextos empresariales que posiblemente ignoren su bienestar.

Ahora bien, en el año 2005 Lene Wendland llegó a la dirección de la Sección de Derechos Humanos y Asuntos Económicos y Sociales, de la Oficina del Alto Comisionado para los Derechos Humanos de las 
Naciones Unidas, y desde allí decidió emprender la construcción de un nuevo rumbo y una mirada más amplia que incluyera el papel de los empresarios en la protección de los derechos humanos. De este modo, Kofi Annan, secretario general de la Organización de las Naciones Unidas (ONU) entre 1997 y 2006, decidió designar a John Ruggie, profesor de la Universidad de Harvard, para el diseño normativo de este nuevo enfoque y su incorporación.

Seguido de esto, tres años después, en el año 2008, el profesor John Ruggie manifestó que emprendería la construcción de unos Principios Rectores sobre empresas y derechos humanos, sustentados principalmente en tres conceptos: proteger, respetar y remediar. El primero pensado en la obligación del Estado en la protección de los derechos humanos; el segundo pensado en términos de la responsabilidad empresarial en el respeto por los derechos humanos en sus acciones; y el tercero visto desde el acceso a vías de reparación para quienes han sido o son víctimas de violaciones a sus derechos humanos en el marco de acciones empresariales (ONU, 2012, p. 1). Con esto determinado, se inició un profundo proceso de investigación que involucró consultas a empresas, gobiernos, sociedad civil, poblaciones y comunidades afectadas, abogados, juristas, inversores y demás involucrados en la puesta en práctica de los principios (ONU, 2012, p. 1).

En 2011 el Consejo de Derechos Humanos de las Naciones Unidas reconoció y se apropió de los Principios Rectores sobre las empresas y los derechos humanos de manera unánime y fueron reconocidos como los Principios Ruggie. Actualmente son un referente del derecho internacional porque involucra el Estado, las empresas y la sociedad civil en responsabilidades y deberes que están en medio de la economía y la actividad comercial. Estos principios están divididos en tres pilares base: proteger, respetar y remediar.

Estos principios fueron consagrados como una norma de conducta a nivel internacional, por lo que las empresas y demás actores involucrados 
deben cumplir. Aunque no tienen un «carácter jurídicamente vinculante, en los Principios Rectores se analizan las repercusiones que las normas y prácticas actuales tienen para los Estados y las empresas y se incluyen algunos aspectos que se contemplan de diversas formas en la legislación nacional e internacional» (ONU, 2012, p. 1). Aunque son principios que hacen parte del soft law, lo que implica una carencia de fuerza vinculante por no estar elevados a la categoría de tratado internacional, adquieren relevancia jurídica debido a que reglamentan, direccionan y dan los lineamientos para las buenas prácticas de protección de los derechos humanos.

El panorama anterior denota la relevancia de estos principios en la defensa de los derechos humanos. En relación con esto, siendo una alternativa novedosa, recientemente las jurisprudencias nacional e internacional han utilizado estos principios, igual que el concepto de debida diligencia para realzar la importancia de los derechos fundamentales de las comunidades étnicas, como es el caso de la consulta previa. Concretamente, sostenemos que los principios Ruggie, por medio de la debida diligencia, refuerzan la garantía y salvaguarda del derecho fundamental a la consulta previa de las comunidades étnicas frente a proyectos, obras o actividades de particulares, así como medidas legislativas o administrativas del Estado que son susceptibles de generar afectaciones directas, y que en ocasiones ven a la consulta como un simple procedimiento, y desvirtúan su naturaleza de derecho fundamental.

Para dar cuenta de lo anterior, se describirá en un primer momento el concepto de debida diligencia en los términos del Estado y la empresa, así como el derecho fundamental de consulta previa. Posteriormente, se presentarán algunos ejemplos nacionales e internacionales que abordan los principios Ruggie, y finalmente, se traerá a colación la sentencia de unificación SU-123 del 2018, decisión base sobre consulta previa en el país, que incorpora los principios Ruggie y la debida diligencia para proteger el derecho fundamental señalado. 


\section{Debida diligencia y consulta previa}

Los Principios Rectores de Naciones Unidas sobre las empresas y los derechos humanos plantean que la debida diligencia «[...] implica un cambio en el enfoque tradicional de las empresas frente a los derechos humanos basado en el conocer y denunciar, por un enfoque de corresponsabilidad donde la empresa asume un rol muy importante de identificar y gestionar» (Consejería Presidencial para los Derechos humanos, 2015 , p. 2). Teniendo en cuenta esta premisa, la labor de identificar y gestionar debe ser asumida tanto por el Estado como por la empresa, y deberá respetar y garantizar la no afectación de los derechos humanos. Se habla de un proceso continuo que las empresas y los Estados llevan a cabo para identificar, prevenir y mitigar los impactos negativos que se presenten en materia de derechos humanos durante el desarrollo o ejecución de las operaciones y los proyectos que se lleven a cabo en un territorio específico.

Ahora bien, la debida diligencia a cargo del Estado se encamina al cumplimiento de la obligación principal que tiene de respetar y proteger los derechos humanos, mediante la supervisión y vigilancia del cumplimiento de los planes de contingencia o precaución que tenga la empresa producto de su propia diligencia, así como velar por que los órganos estatales actúen de forma coherente con las buenas prácticas empresariales. Esta debida diligencia se encuentra consagrada en los tres Principios Rectores de Naciones Unidas (PRNU) que establecen que:

1. Los Estados, en cumplimiento de su obligación de protección, deben asesorar de manera eficaz a las empresas sobre cómo respetar los DDHH en sus actividades, incluido el ejercicio de la debida diligencia en DDHH (Principio $n^{\circ}$. 3) (ONU, 2011, p. 5).

2. Los Estados deben exigir a las empresas de su propiedad o bajo su control realizar la debida diligencia en materia DDHH en el marco 
de sus operaciones y de su relacionamiento con terceros (Principio $n^{\circ}$. 4) (ONU, 2011, p. 7).

3. Las empresas deben proceder con debida diligencia para responder a su deber de respeto de los DDHH con el fin de identificar, prevenir, mitigar y responder frente a las consecuencias negativas de sus actividades sobre los derechos de otros (Principio $n^{\circ}$. 17) (ONU, 2011, p. 17).

En el marco de los Principios Rectores, se destacan como elementos que debe contener la aplicación de la debida diligencia los siguientes: compromiso de la empresa frente al respeto de los derechos humanos (Principios 16 y 21); evaluación periódica que evidencie los impactos reales y potenciales que se presenten en el desarrollo de las actividades propias de la empresa y su relación con los derechos humanos (principio 18); integración de los compromisos adquiridos y 1 as evaluaciones de los sistemas de control internos (Principio 19); y finalmente, se establece la realización de un seguimiento que deberá estar acompañado de la presentación de informes de desempeño, de acuerdo con el Principio 20 (ONU, 2011).

Lo expuesto se enmarca en la responsabilidad directa que tiene y debe tener la empresa de proteger a las comunidades a través de garantías para el goce de sus derechos, lo que supone que se encuentra en el marco de protección, respeto y reparación. En la práctica, esto se evidencia en las formas en las que una empresa es consciente de un riesgo creado, evita ese riesgo y lo enfrenta mediante la implementación de políticas, procesos de evaluación de riesgos e impactos y sistemas de gestión adecuados.

Vale la pena resaltar que la debida diligencia debe ser previa a la ejecución de políticas y planes de desarrollo económico, ya que solo así se logran identificar problemas futuros para los que se debe establecer 
planes de contingencia que deberán ser aplicados al momento de generarse una afectación a los derechos humanos.

Sin que se considere menos relevante, y después de haber abordado el concepto de debida diligencia, la consulta previa libre e informada se define como un derecho fundamental de los grupos étnicos, que resulta exigible cuando el Estado origina medidas legislativas, administrativas, o cuando los particulares construyen un proyecto, obra o actividad que puede generar modificaciones o afectaciones directas en las comunidades étnicas. Este derecho fundamental constituye un diálogo horizontal entre comunidades étnicas, Estado y particulares.

Sumado a lo anterior, el Convenio 169 de la Organización Internacional del Trabajo (OIT) es el tratado internacional que incorpora la consulta previa en el país. Concretamente, fue ratificado por Colombia a través de la Ley 21 de 1991 -integrándose al bloque de constitucionalidady establece las bases necesarias para garantizar la consulta previa de las comunidades étnicas. Así mismo, ha reforzado la autonomía de las comunidades en sus territorios, pues estas últimas tienen el derecho a participar en el proceso de toma de decisiones que los puede afectar directamente.

Por último, la Directiva 10 del 2013, de la Presidencia de la República de Colombia, aclara el orden del procedimiento y las etapas de la consulta previa, y la Directiva 01 del 2010 especifica las acciones que requieren o no consulta previa.

Todas estas fuentes nacionales e internacionales garantizan el reconocimiento expreso de principios constitucionales como la participación y el pluralismo (Artículo $1^{\circ}$ ); la diversidad étnica y cultural (Artículo $7^{\circ}$ ); la igualdad general, material y de culturas (Artículos 13 y 70); el derecho a la participación ciudadana (Artículo 40, numeral 2), y la obligación estatal de participación de comunidades indígenas (artículo 330). 


\section{La articulación de los principios Ruggie, la debida diligencia y la consulta previa}

Existen casos nacionales e internacionales que hacen mención a la violación o protección de los derechos humanos. En el ámbito internacional se trae a colación los casos de las empresas Royal Dutch Shell, junto a Chevrón, BP [...] que participan en un proyecto de extracción de gas y petróleo en la Patagonia-Argentina, que está afectando los derechos de territorio de comunidades indígenas, así como los de medioambiente (Álvarez, 2018, párr.6), y las empresas Iberdrola, Gas Natural Fenosa, Acciona [...] que participaron en el corredor eólico de América Latina, y que generaron impactos en el territorio de varias comunidades, de acuerdo con el Observatorio de Multinacionales en América Latina, que describe que se han utilizado medios ilegales para modificar la propiedad de la tierra, así como varios impactos ambientales (Álvarez, 2018, párr. 6).

Por su parte, en el ámbito nacional se destacan los casos de Repsol en la alta Guajira, "proyecto de sísmica en 2D en la Península de la Guajira en Colombia, donde se llevó a cabo una evaluación de impacto en derechos humanos entre finales de 2014 y principios de 2015, como parte del proceso de debida diligencia en derechos humanos» (Repsol, s.f, párr. 1); la Sentencia T-732 del 2016, que conoció de una familia que decidió interponer una acción de tutela contra el Oleoducto Central S.A y el Consorcio de Tierras, a quienes consideraron responsables del deterioro de su vivienda por el tránsito de maquinaria pesada por el proyecto de construcción de una estación de bombeo de petróleo en el municipio de Páez, Boyacá; y el reconocido caso de Drummond por su participación en el conflicto armado colombiano, financiando actividades de grupos paramilitares (Business \& Human Rigths Resource, 2018).

Como puede observarse, ninguno de los casos anteriores presenta una relación entre el derecho de consulta previa y los principios Ruggie; solo se refieren en estricto sentido a los segundos. Luego de hacer la búsqueda, 
encontramos que, en el ámbito internacional, se registra en la Corte Interamericana de Derechos Humanos (CIDH) una decisión del año 2015 — caso de Pueblos Kaliña y Lokono vs. Surinam-, que analiza la responsabilidad internacional del Estado de Surinam por una serie de violaciones de los derechos de los miembros de ocho comunidades de los pueblos indígenas Kaliña y Lokono del Río Bajo Marowijne. Entre los diversos argumentos, se señala que el otorgamiento de concesiones, licencias mineras y el establecimiento y permanencia de reservas naturales no han sido sometidos al procedimiento de consulta dirigido a obtener el consentimiento previo, libre e informado de los pueblos. De lo anterior la $\mathrm{CIDH}$ encontró que las actividades mineras que generaron afectaciones al medioambiente y a los derechos de los pueblos indígenas, fueron llevadas a cabo por actores privados (empresas). Acerca de este punto, toma como referencia los Principios Rectores sobre las empresas y los derechos humanos, avalados por el Consejo de Derechos Humanos de las Naciones Unidas, que establecen que las empresas deben actuar de conformidad con el respeto y la protección de los derechos humanos, así como prevenir, mitigar y hacerse responsables por las consecuencias negativas de sus actividades sobre los derechos humanos.

En conclusión, determinó que el Estado no garantizó la realización de un estudio de impacto ambiental y social de manera independiente y previa al inicio de la extracción de los recursos, ni supervisó el estudio que fue realizado con posterioridad, teniendo en cuenta que es un área natural protegida y de territorios tradicionales para diversos pueblos.

En contraste, a nivel nacional, la sentencia de unificación de la Corte Constitucional SU-123 del 2018, que se abordará a continuación, es la única decisión que analiza los principios Ruggie a la luz de la consulta previa a comunidades étnicas; una situación alentadora y fundamental para la protección de este derecho fundamental.

Los casos presentados dan cuenta de la escasa articulación que se ha efectuado alrededor del derecho fundamental a la consulta previa y los 
principios Ruggie con la debida diligencia, situación que acentúa la necesidad de continuar fortaleciendo esta relación con el objetivo de garantizar la aplicación del derecho fundamental de consulta previa, el cual, en el caso colombiano, no se ha cumplido siempre a cabalidad por parte de las empresas ni el Estado.

\section{La Sentencia SU-123 del 2018}

Una decisión novedosa en torno a la garantía y defensa del derecho a la consulta previa en Colombia fue la reciente Sentencia SU-123 del 2018, que abordó la acción de tutela presentada por un representante del cabildo indígena awá «La Cabaña», quien solicitó la protección de los derechos fundamentales a la consulta previa y el ambiente sano de la comunidad. Este grupo, que habita en Putumayo, se vio afectado ambientalmente por una exploración petrolera que se adelantó en el territorio de su cabildo, y donde nunca se adelantó consulta previa. Los derechos fundamentales señalados fueron amparados por la Corte Constitucional.

En este fallo, además de recordarse todas las fuentes nacionales e internacionales que defienden el derecho fundamental de consulta previa en el país, se agrega un nuevo elemento que llama mucho la atención, y que pertenece a la línea de derechos humanos y empresa: los principios Ruggie. Al respecto, la sentencia señala que a pesar de que el deber principal de proteger los derechos fundamentales de todas las personas, incluida la población indígena, recae en el Estado, las empresas también tienen deberes de respeto de los derechos humanos y un deber de debida diligencia frente a la consulta previa de los pueblos indígenas y las comunidades afrodescendientes (Corte Constitucional, Sentencia SU-123).

Lo anterior denota una gran importancia, pues la exigencia de garantía del derecho fundamental de consulta previa ya no proviene solamente del Convenio 169 de la OIT, la Constitución Política, la jurisprudencia 
de la Corte Constitucional, y las directivas 10 del 2013 y 1 del 2010, sino que se suma otro instrumento más como los principios Ruggie, que a pesar de ser soft law, como se mencionó anteriormente, son un instrumento clave para la consulta previa.

La Corte Constitucional considera que, en los casos de consulta previa, los principios Ruggie son referentes para valorar la actuación de las empresas con respecto del deber de desarrollar la consulta previa, pues como lo ha señalado la Corporación, «los pronunciamientos de las instancias internacionales, encargadas de interpretar los diferentes instrumentos del derecho internacional de los derechos humanos, constituyen un criterio hermenéutico relevante para establecer el sentido de las normas constitucionales sobre derechos fundamentales» (Sentencia SU-123). También, considera que utilizar el estándar de debida diligencia de las empresas en relación con el respeto de los derechos de las comunidades étnicas permite determinar si se vulneran derechos de una comunidad étnicamente diferenciada. La debida diligencia sirve para que las autoridades, en un eventual ejercicio de ponderación, puedan determinar cuáles han sido ejercidos legítimamente. Así mismo, los parámetros de debida diligencia permiten adecuar las actuaciones de las autoridades públicas y de los particulares a los principios constitucionales de buena fe (artículo 83, C.P.) y confianza legítima, toda vez que otorgan estabilidad a los actores involucrados en relación con los lineamientos y criterios que deben seguir para cumplir con los mandatos de la consulta previa.

Con esto presente, y trayendo el caso concreto colombiano relacionado con la consulta previa, el argumento de las empresas no puede ser que el Estado (a través del Ministerio del Interior) expide una certificación de no presencia de comunidades étnicas, y que este documento es su respaldo. Igualmente, tampoco podrá argumentar que la responsabilidad directa es del Estado. Por lo tanto, hay un deber de diligencia de las organizaciones en detectar previo al desarrollo de los proyectos o actividades que van a desarrollar, posibles afectaciones directas que se 
puedan generar a las comunidades étnicas, que como se ha reiterado a través de la jurisprudencia de la Corte Constitucional,

la protección de los valores culturales, económicos y sociales de los pueblos indígenas y tribales, que aún subsisten en el territorio nacional, es un asunto de interés general en cuanto comporta el reconocimiento de la diversidad étnica y cultural de la nación colombiana, y la existencia misma del Estado social de derecho. (Sentencia SU-383, p. 123)

De esta manera, se puede argüir que los principios Ruggie - como instrumento jurídico - refuerzan la protección de los valores culturales, económicos y sociales de los pueblos indígenas y tribales, quienes son los sujetos principales del derecho fundamental a la consulta previa; pero también protegen a las comunidades no étnicas en los contextos de actividad empresarial. A su vez, estos principios son una herramienta importante para fomentar la relación armónica entre el Estado, los particulares y las comunidades étnicas y, además, permiten entender que las empresas tienen un deber de respeto en las actuaciones que adelantan con los derechos humanos. Los pronunciamientos efectuados en la decisión dan cuenta de la necesidad de la Corte Constitucional de reconocer las debilidades del Estado, y exigir el cumplimiento de sus obligaciones, pero también «ataca» una falacia recurrente en la omisión del derecho fundamental a la consulta previa por parte de las empresas, como lo es el valerse de la ineficacia institucional, al recordarles las responsabilidades que tienen en su operación, conforme a lo manifestado en los principios Ruggie.

\section{Conclusiones}

Es importante resaltar que estos principios exponen la responsabilidad que tiene cada Estado de proteger a las personas dentro de su territorio contra las violaciones de derechos humanos (un deber que proviene del derecho internacional). También recuerda que los Estados miembros de las Naciones Unidas deben resaltar la responsabilidad que tienen las 
empresas de actuar en los términos de los derechos humanos, esto es, no causar daño, y tomar las precauciones necesarias y razonables para prevenir impactos negativos en el desarrollo de su operación. A su vez, esto es absolutamente necesario porque durante las últimas décadas el fuerte proceso de expansión económica en América Latina y Colombia, ha generado la apertura del mercado y la llegada de empresas en busca de materias primas; muchos de estos productos son recursos naturales ubicados en territorios rurales donde habitan comunidades étnicas y no étnicas, que de no tomar precauciones pueden transformar su permanencia en estos espacios.

Así mismo, los principios Ruggie son un instrumento jurídico que, aunque no es vinculante, opera como mediador entre las empresas y las comunidades. De la misma manera, es de suma importancia el reconocimiento del sector empresarial como un actor que tiene deberes y responsabilidades en la protección de los derechos humanos, los cuales se ven plasmados en el proceso de debida diligencia, como se explicó anteriormente. Por último, se concluye que la debida diligencia es una herramienta que ayuda a la empresa y al Estado a determinar un modo de acción para evitar que en el futuro tenga que reparar y remediar violaciones a derechos humanos; así mismo, la debida diligencia es el mecanismo para ejecutar y poner en práctica los PRNU.

No hay que perder de vista que este reconocimiento evita que las empresas utilicen justificaciones, que relacionan la ausencia del Estado y la ineficacia institucional, para desestimar las violaciones a derechos humanos que se puedan cometer.

\section{Referencias}

Álvarez, Yago (16 de octubre de 2018). Cinco violaciones de derechos humanos por multinacionales europeas que hacen necesario un tratado vinculante. Comité para la abolición de las deudas ilegítimas. Recuperado de: https:// bit.ly/2m2tSR6. 
Business \& Human Rigths Resource (2018). Colombia: Fiscalía señala a funcionarios de Drummond por financiar crímenes de guerra paramilitares de 1996 a 2006; con comentarios de la empresa [Comunicado]. Recuperado de https://bit.ly/2lBhZS1.

Calvo, José (7 de junio de 1996). Acusan a Nike de fabricar calzado de lujo explotando a niños de 11 años. El País. Recuperado de: https://bit.ly/2v2e3hr.

Corte Interamericana de Derechos Humanos (25 de noviembre de 2015). Pueblos Kaliña y Lokono vs. Surinam. [Fondo, Reparaciones y Costas]. Recuperado de: https://bit.ly/2H6vlOa.

Corte Constitucional de Colombia (Dirección) (2018). Rueda de prensa, SU-123 de 2018. Decisión sobre derechos amparados a comunidad Awá "La Caba$\tilde{n} a ”$. Recuperado de: https://bit.ly/2z9hcv8.

Consejería Presidencial para los Derechos Humanos (2015). Colombia Avanza. Derechos Humanos y Empresa. Plan de Acción de Derechos Humanos y Empresa. Bogotá: Gobierno nacional.

Hurtado, Carlos (2011). Empresas y derechos humanos: hacia el desarrollo de un marco normativo internacional. Aportes DPLF. Revista de la Fundación para el Debido Proceso, 15(4), 7-11.

I Arrufat, Jordi (3 de diciembre de 2014). Bhopal, una noche que dura ya 30 años. El País. Recuperado de https://bit.ly/2P1MNtq.

Organización de las Naciones Unidas [ONU] (2011). Principios rectores sobre las empresas y los derechos humanos. Nueva York y Ginebra: ONU.

Organización de las Naciones Unidas [ONU] (2012). La responsabilidad de las empresas de respetar los derechos humanos. Guía para la interpretación. Nueva York y Ginebra: ONU.

Repsol (S.f). Derechos Humanos: Estudio de Impacto en Derechos Humanos Alta Guajira. Recuperado de: https://bit.ly/2kutyKk.

Slack, Keith (2011). Derechos humanos e industrias extractivas en América Latina. Aportes DPLF. Revista de la Fundación para el Debido Proceso, 15(4), 4-6.

Corte Constitucional (13 de mayo de 2003). Sentencia SU-383. [MP Álvaro Tafur Galvis].

Corte Constitucional (15 de noviembre de 2018). Sentencia SU-123. [MP Alberto Rojas y M. P. Rodrigo Uprimny]. 